\author{
A. van Veen \\ A. Karstens \\ A. C. J. van der Hoek \\ D. Tibboel \\ K. Hählen \\ E. van der Voort
}

\section{The prognosis of oncologic patients in the pediatric intensive care unit}

Received: 1 February 1994 Accepted: 14 July 1995
E. van der Voort ( $x-2)$

Department of Pediatrics,

Division of Pediatric Intensive Care.

Sophia Children's Hospital,

Dr. Molewaterplein 60 .

NL-3015 GJ Rotterdam, The Netherlands

A. van Veen - A. Karstens A.C.J. van der Hock Erasmus University, Rotterdam,

The Netherlands

D. Tibboel

Department of Pediatric Surgery,

Surgical Intensive Care Unit,

Sophia Children's Hospital,

Rotterdam, The Netherlands

K. Hählen

Department of Pediatries,

Division of Hematology/Oncology,

Sophia Children's Hospital,

Rotterdam, The Netherlands
Abstract Objective: To evaluate the predicted mortality rate of oncologic patients in the PICU using the PRISM score and factors that might influence short-term outcomes.

Design: Retrospective study.

Setting: Pedriatic ICU in a university hospital.

Patients and Methods: The medical charts of all oncologic patients admitled to the PICU during the period from January 1983 to December 1992 were revicwed.

Main Results: Over a period of 10 years, 51 oncologic pationts were admitted on 57 occasions to the PICU. The mortality was $32 \%$. This is significantly higher than the overall mortality in the PICU $(8 \%)$. Comparison of observed and predicted mortality, derived from the PRISM score, using chi square goodness-of-fit tests showed a significantly higher observed mortality $\left(x^{2}(5)=20.1, P<0.01\right)$. Patients admitted for circulatory failure had the highest mortality (47\%), followed by those with respiratory failure due to tachypnea/cyanosis $(36 \%)$, central nervous system deterioration $(27 \%)$, respiratory failure due to airway obstruction $(25 \%)$, and metabolic disorders $(20 \%)$. Of the 31 patients who needed mechanical ventilation, 17 died $(55 \%)$, and when they needed inotropic support as well, the mortality increased to $69 \%$. The mortality rose to $100 \%$ when the patient was admitted with a septic shock, necessitating mechanical ventilation and inotropic support. The median PRISM score was 5 in the survivor group and 18.5 in the non-survivor group; this difference was found to be significant using the Wilcoxon test $(P=0.01)$. However, some patients with high scores were found in the survivor group, as well as some with low scores in the non-survivor group.

Conclusion: The decision to treat oncologic patients in a PICU remains difficult and has to be considered on an individual basis. However, oncologic patients do benefit from admission to the PICU. The PRISM score is not suitable for oncologic patients in the PICU, because it underestimates the observed mortality. Other factors like neutropenia, septic shock, the need for mechanical ventilation, and inotropic support should be taken into consideration.

Key words Children - Malignancy Prognosis - Intensive care PRISM score 


\section{Introduction}

Children with a malignancy have a relatively good prognosis in comparison with adults. In pediatric oncology, an overall 5-year survival rate of $66.8 \%$ has been reached [1]. However, treatment of oncologic patients is very intensive and life-threatening complications can occur. These complications, such as respiratory failure, circulatory failure, neutropenia, and septic shock, may require treatment in a pediatric intensive care unit (PICU). The decision to admit oncologic patients to the PICU may be difficult, not because the outcome from their underlying disease is uncertain, but because the results of PICU admission and therapy in terms of futile suffering, efforts, and costs might be discouraging for patients, parents, and doctors. Knowledge of prognostic factors and outcomes of oncologic patients in the PICU is essential. To assess severity of illness and to calculate the mortality risk, scoring systems can be used. We used the pediatric risk score for mortality (PRISM) to assess the severity of illness at admission. This score matches the observed mortality very well in non-oncologic patients [2]. Oncologic patients admitted to the PICU have a mortality rate far above the overall mortality $[3,4,5]$. One might speculate that the PRISM score in its unadapted form is not as suitable for oncologic as for non-oncologic patients.

This study was undertaken: (1) to evaluate the outcome of oncologic patients in the PICU, (2) to evaluate the PRISM score in oncologic patients, and (3) to identify other factors that might influence short-term outcomes.

\section{Patients and methods}

The Sophia Children's Hospital in Rotterdam is one of the four pediatric oncology centers in the Netherlands. About 65 new oncologic patients are admitted each year. The medical charts of all oncologic patients admitted to the PICU over a 10 -year period (January 1983-December 1992), were reviewed. The admission policy for oncologic patients is the same as for other patients. However, in oncologic patients who are clearly in the final stage of their oncologic disease, a "do not resuscitate" policy is often used and therefore these patients are not admitted to the PICU when they need vital support. Therefore, this group was not included in this study. When a patient was admitted to the PICU more than once, all admissions were taken into account. Patients admitted for postoperative surveillance were also excluded from this study

The information retrieved from the medical charts included: age, sex, oncologic diagnosis, date of diagnosis, chemo- or radiotherapy in previous treatment regimens, operation before admission, cause of admission, duration of stay, neutropenia, infection, septic shock, respiratory infection, respiratory failure, mechanical ventilation, inotropic support, survival, and eventual cause of death. Table 1 shows the number of patients admitted according to cause of admission.

To diagnose septic shock, at least four of the following criteria had to be met [6]: (1) body temperature $<35.5^{\circ} \mathrm{C}$ or $>38.9^{\circ} \mathrm{C}$, (2) hypotension (systolic pressure $<5$ th percentile for age), (3) tachypnea ( $>95$ th percentile) or hypocapnia $\left(P_{\mathrm{a}} \mathrm{CO}_{2}<4.3 \mathrm{kPa}\right.$ ), (4) tachycardia (heart rate $>95$ th percentile), (5) abnormal white blood-cell count $\left(<3.5^{*} 10^{9} / 1\right.$ or $\left.>15.0^{*} 10^{9} / 1\right)$ or a severe shift to the left, (6) thrombocytopenia $\left(<100^{*} 10^{9} / 1\right)$, (7) surgical or invasive procedure performed during the previous $48 \mathrm{~h}$ or the presence of an obvious primary septical site.

To diagnose respiratory infection, not only an abnormality on the chest radiograph had to be found, but two of the following items as well: (1) a positive sputum culture, (2) a body temperature $>38.5^{\circ} \mathrm{C}$ or (3) an elevated ESR or CRP.

The diagnosis of respiratory failure was made, when the following criteria were found [7]: (1) a $P_{\mathrm{a}} \mathrm{O}_{2}<10 \mathrm{kPa}$ with an $\mathrm{FiO}_{2}>0.5$, (2) diffuse bilateral pulmonary infiltrates on chest radiograph, (3) exclusion of cardiogenic pulmonary edema clinically or with pulmonary artery catheterization $(\mathrm{PAOP}<2.9 \mathrm{kPa}$ ).

A patient was considered neutropenic if a neutrophil count of $<1.0^{*} 10^{\circ} / 1$ was found. We divided this group into subgroups, one group with neutrophil counts of $<1.0$ and the other with $<0.1 * 10^{9} / 1$

In order to assess objectively the severity of illness the PRISM score, a scoring system using 14 physiological variables, was applied. The PRISM score was measured on the day of admission and the mortality risk was calculated using the algorithm of Pollack et al. [2]. To calculate the mortality risk of a group, the mean of all individual mortality risks was used.

PICU mortality was defined as death in the PICU. Whenever a "not to be resuscitated" (NTBR) policy was used in the PICU it was on the basis of the current disease, not on the basis of the oncologic disease. These patients died in the PICU (PICU mortality).

To compare predicted and observed mortality, the chi square goodness-of-fit test was used. The Wilcoxon test was used to compare PRISM scores in the survivor and non-survivor groups.
Table 1 PRISM score, predicted and observed mortality according to causes of admission

\begin{tabular}{lcllccc}
\hline $\begin{array}{l}\text { Cause of } \\
\text { admission }\end{array}$ & $\begin{array}{l}\text { Number of } \\
\text { patients }\end{array}$ & $\begin{array}{l}\text { Inotropic } \\
\text { support }\end{array}$ & $\begin{array}{l}\text { Septic } \\
\text { shock }\end{array}$ & $\begin{array}{c}\text { Median } \\
\text { PRISM }\end{array}$ & $\begin{array}{l}\text { Mortality } \\
\text { predicted }\end{array}$ & $\begin{array}{l}\text { Mortality } \\
\text { observed }\end{array}$ \\
\hline $\begin{array}{l}\text { Respiratory failure } \\
\text { Airway obstruction }\end{array}$ & 4 & $1(25 \%)$ & $0(0 \%)$ & $10.5(2-55)$ & $29 \%$ & $25 \%$ \\
Tachypnea/cyanosis & 14 & $7(50 \%)$ & $1(7 \%)$ & $9.0(0-26)$ & $12 \%$ & $36 \%$ \\
Neurological & 15 & $2(13 \%)$ & $0(0 \%)$ & $10.0(0-42)$ & $19 \%$ & $27 \%$ \\
Metabolic & 5 & $0(0 \%)$ & $2(40 \%)$ & $12.0(5-14)$ & $7 \%$ & $20 \%$ \\
Circulatory failure & 15 & $8(53 \%)$ & $5(33 \%)$ & $7.0(2-26)$ & $10 \%$ & $47 \%$ \\
Miscellaneous & 4 & $0(0 \%)$ & $0(0 \%)$ & $6.0(4-9)$ & $2 \%$ & $0 \%$ \\
\hline
\end{tabular}




\section{Results}

A total of 51 patients were admitted on 57 occasions. The group consisted of 31 boys and 26 girls, the median age was 6.9 years (range $0-17$ ). The mortality of this group of patients was $32 \%(18 / 57)$. The overall mortality in the PICU evaluated over the study period was $8 \%$. The number of patients with inotropic support and septic shock, the median PRISM scores and the predicted and observed mortality rate of each admission category are listed in Table 1.

Compared to the observed mortality, the predicted mortality, based on the PRISM score $(13 \%)$, is significantly lower $\left(x^{2}(5)=20.1, P<0.01\right)$. Table 2 compares the survivor and non-survivor groups and shows significantly differences between the median PRISM scores: 18.5 (6-55) for the non-survivor group and $5(0-17)$ for the survivor group $(P<0.01)$. However, very low scores can be found in the non-survivor group, and high scores can be found in the survivor group. Mortality rate by PRISM score is shown in Table 3. Except for the group with PRISM score 0 5, the predicted mortality is much lower than the observed mortality.

Important factors related to mortality are septic shock, neutropenia, the need for mechanical ventilation and/or inotropic support (Table 1). In the group of respiratory failure $(n=18) 6$ patients died. All six required mechanical ventilation and 4 needed inotropic support as well. In the circulatory failure group $(n=15) 6$ of the 7 patients who died required mechanical ventilation and inotropic support, and 3 of them suffered from a septic shock as well. Of the patients admitted for neurological causes $(n=15) 4$ died. Two of these patients needed mechanical ventilation and two needed inotropic support and mechanical ventilation. The non-surviving patient in the metabolic group, admitted for serious electrolyte disturbances leading to rhythm disturbances, was also suffering from septic shock and required mechanical ventilation.

Table 2 Differences between the survivor and non-survivor groups

\begin{tabular}{|c|c|c|c|}
\hline & $\begin{array}{l}\text { Non-survivors } \\
n=18\end{array}$ & $\begin{array}{l}\text { Survivors } \\
n=39\end{array}$ & Mortality \\
\hline Artificial ventilation & $17(94 \%)$ & $14(36 \%)$ & $17 / 31(55 \%)$ \\
\hline Inotropic support & $11(61 \%)$ & $7(18 \%)$ & $11 / 18(61 \%)$ \\
\hline $\begin{array}{l}\text { Ventilation and } \\
\text { inotropes }\end{array}$ & $11(61 \%)$ & $5(13 \%)$ & $11 / 16(69 \%)$ \\
\hline $\begin{array}{l}\text { Ventilation, inotropes } \\
\text { and septic shock }\end{array}$ & $3(17 \%)$ & $0(0 \%)$ & $3 / 3(100 \%)$ \\
\hline Neutropenia & $7(39 \%)$ & $8(21 \%)$ & $7 / 15(47 \%)$ \\
\hline
\end{tabular}

Of the 31 patients who were mechanically ventilated $55 \%$ of them died; 16 patients received both mechanical ventilation and inotropic support and 69\% of them died. Three patients were in septic shock and needed mechanical ventilation and inotropic support; their mortality was $100 \%$. Of the 15 neutropenic patients 7 died $(47 \%)$. Eight neutropenic patients needed mechanical ventilation and $7(88 \%)$ of them died (Table 2).

The median duration of stay in the PICU was not significantly different for survivors; 5 (1-59) days, as compared to non-survivors; 2 (1-19) days.

\section{Discussion}

This study was undertaken to investigate the outcome and predictability of outcome of oncologic patients in the PICU. In general patients are admitted when invasive monitoring is required or when they need support of the vital functions. We found a $32 \%$ mortality rate in this group of oncologic patients, which is much higher than the overall mortality rate $(8 \%)$ in the PICU. In the literature on oncologic patients, PICU mortalities of $51 \%$ [3], 48\% [4] and 49\% [5] were found.

Major differences in mortality rates are found when the groups are subdivided according to admission criteria. The 15 patients admitted for circulatory failure had the worst outcome with a mortality rate of $47 \%$. Sivan et al. [3] also found a mortality rate of $47 \%$ in this group, while Butt et al. [4] found a much higher mortality rate of $75 \%$.

The PICU mortality in the group of patients with respiratory failure due to tachypnea/cyanosis was $36 \%$. In the other studies, mortality rates of $75 \%$ [4], 73\% [5] and 60\% [3] were found. The discrepancy increases in the group with impaired ventilation due to tumormass effects: in this group mortalities of $0 \%$ [4], 20\% [5] and 75\% [3] were reported. In our group of 4 patients, 1 patient died $(25 \%)$.

Another large difference is found in the group of patients admitted with primarily neurological disease. We found a mortality of $27 \%(4 / 15)$, while others found a 38\% [5], 48\% [4] and $90 \%$ [3] mortality. Three of the 4 non-survivors in this group died on the day that the diagnosis of leukemia was made. All 3 suffered from cerebral bleeding and died of cerebral compression resulting in brain death.

In all of our subgroups and in all of the studies on this subject, the mortality of ventilated patients is higher than that of non-ventilated patients $\left(x^{2}(5)=14.2\right.$, $P<0.05)$. Twenty-six children $(46 \%)$ did not require mechanical ventilation and $1(3 \%)$ of them died. Of our 
31 ventilated patients, $17(55 \%)$ died (Table 2). However, part of the ventilated patients required inotropic support as well or were in a septic shock. There were 12 patients who needed mechanical ventilation without inotropic support and who were not in shock. Of these 12 patients 4 died $(33 \%)$. Mortality rises to $62 \%$ when there is a need for inotropic support and mechanical ventilation and finally a $100 \%$ mortality is found when the patients also suffered from a septic shock.

We, as in the other studies, found a very high mortality in the group of patients suffering from a septic shock: five out of eight patients died $(63 \%)$. Two of these non-surviving patients were not neutropenic but they did need mechanical ventilation. The three survivors were neutropenic but did not need artificial ventilation or inotropic support.

Fifteen patients were neutropenic and 7 of them died (47\%). Sivan et al. [3] concluded that the severity of the neutropenia does affect the prognosis. In our study, seven patients had a neutrophil count of $<0.1 * 10^{9} / 1$, four of whom died in the PICU, while eight patients had a neutrophil count of $0.1-1.0 * 10^{9} / 1$, three of them died in the PICU. Although we suspect the severity of neutropenia to be an important prognostic factor, we cannot draw that conclusion because the groups are too small. Another factor that could affect outcome is whether or not there is a rise in the neutrophil count. In none of the non-survivors was a rise in the count seen. However, after one week the neutrophil count had risen in only three of the eight survivors. Again, no conclusion can be drawn from these small numbers.

The PRISM score is used to asses the severity of illness and to help predict the short-term outcome in groups of patients. However, the PRISM score cannot be used to predict mortality of an individual patient, because there is an overlap in the scores of survivors and those of non-survivors. Our results show that the PRISM score is not useful in predicting mortality for oncologic patients. Although the observed mortality rises with higher PRISM scores, we found that the predicted mortality was significantly lower than the observed mortality $\left(x^{2}(5)=20.1, P<0.01\right)$. Dividing the group of patients into subgroups according to their PRISM scores, this difference was seen in all groups except in the groups with a PRISM score of $0-5$ and $>25$ (Table 3 ). We found that the predicted mortality was too low in the groups where for admission was metabolic, cardiogenic, or respiratory failure due to tachypnea/cyanosis (Table 1).

The mortality rate of the oncologic patients in the PICU in our study $(32 \%)$ is much lower than that found in the other studies, which were all approxi-
Table 3 Mortality rate by PRISM score

\begin{tabular}{clcc}
\hline PRISM score & $\begin{array}{l}\text { Number of } \\
\text { patients }\end{array}$ & $\begin{array}{l}\text { Predicted } \\
\text { mortality }\end{array}$ & $\begin{array}{c}\text { Observed } \\
\text { mortality }\end{array}$ \\
\hline $0-5$ & 20 & $1 \%$ & $0 \%$ \\
$6-10$ & 14 & $3 \%$ & $36 \%$ \\
$10-15$ & 8 & $8 \%$ & $25 \%$ \\
$16-20$ & 7 & $17 \%$ & $57 \%$ \\
$21-25$ & 3 & $42 \%$ & $100 \%$ \\
$>25$ & 5 & $75 \%$ & $80 \%$ \\
\hline
\end{tabular}

mately $50 \%$. Because the overall PICU mortality is the same in all studies $(9 \%$ [4], 8\% [3], 8\% this study), this cannot be explained by differences in quality of care in the PICU's. One possible explanation could be that our patients were not as severely ill as the patients in the other studies. However, the PRISM scores in our series (0-55, median 9), do not differ from the physiologic stability index (PSI) scores in the study of Sivan et al. [3] (1-31, median 11.4). Because the PRISM score is derived from the PSI score, these scores are comparable to one another. Therefore this excludes the possibility that the patients in our study were less severely ill than those in the Sivan et al. study. Heney et al. [5] used the APACHE-II score and in the study of Butt et al. [4] no scoring system was used.

Another possible explanation for the lower mortality found in our study is that in 11 of our 31 ventilated patients, there were no incidents respiratory failure according to the previously described criteria used in this study. The mortality in this group of ventilated patients was $45 \%(5 / 11)$ as compared to a mortality of $60 \%(12 / 20)$ in the group with respiratory failure. In the other studies there are no data on this, but it is possible that our patients were ventilated in an earlier stage.

The difference in mortality rates is probably be due to differences in admission policies. In the period of this study, 48 oncologic patients died in our hospital in wards other than the PICU. In 25 of these patients $(52 \%)$, a NTBR policy was used, because of their poor oncologic prognosis. One hundred-eighteen patients died at home and most of them after a palliative treatment. An explanation for the difference in mortality could therefore be, that in the other studies more endstage patients were admitted to the PICU.

We conclude that children with cancer have a high risk of dying when they reach the stage that admission to the PICU is necessary. The PRISM scoring system is not applicable to this group of patients, therefore a mortality risk cannot be predicted. Mortality rises when the number of interventions such as mechanical ventilation or inotropic support rises, or when complications such as neutropenia or septic shock are 
involved. The question, therefore, rises whether early PICU admission would favorably affect the outcome. In our hospital there is a close cooperation between pediatric oncology and intensive care teams. Good communication is necessary to decide whether a patient should be admitted to the PICU. The decision to continue treatment should not be everlasting; reconsideration has to take place with every major change in therapy or the condition of the patient.

Acknowledgements We would like to thank J.A. Hazelzet for reviewing this paper and D. Shackleton for his help in setting up this study.

\section{References}

1. Gloeckler Ries LA, Hankey BF, Miller BA (1991) Cancer Statistics Review 1973-1988 I. 16

2. Pollack MM, Ruttimann UE, Getson PR (1988) Pediatric risk of mortality (PRISM) score. Crit Care Med 16: 1110-1116

3. Sivan Y, Schwartz PH, Schonfeld T, Cohen IJ, Newth CJL (1991) Outcome of oncology patients in the pediatric intensive care unit. Intensive Care Med 17: $11-15$
4. Butt W, Barker G, Walker C, Gillis J, Kilham H, Stevens M (1988) Outcome of children with hematologic malignancy who are admitted to an intensive care unit. Crit Care Med 16: 761-764

5. Heney D. Lewis IJ, Lockwood L, Cohen AT, Baily CC (1992) The intensive care unit in pediatric oncology. Arch Dis Child 67:294 298
6. Hinshaw L (1987): Effect of high-dose glucocorticoid therapy on mortality in patients with clinical signs of systemic sepsis. N Engl J Med 317: 659-665

7. De Bruin W, Notterman DA, Magid M, Godwin T, Johnston S (1992) Acute hypoxemic respiratory failure in infants and children: clinical and phathologic characteristics. Crit Care Med 20: 1223-1234 